\title{
Research on Factors Affecting Knowledge Transfer in Mentoring Process
}

\author{
Li Nan \\ Professor, College of Economics and Management, \\ Nanjing University of Aeronautics and Astronautics, Nanjing, China
}

Guo Hui

Postgraduate Student, College of Economics and Management, Nanjing University of Aeronautics and Astronautics, Nanjing, China

Shi Yang

Lecturer, College of Management, Changshu Institute of Technology Changshu, China

Lv Lizhi

Professor of the Institute of Higher Education, Nanjing University of Aeronautics and Astronautics

\section{Doi:10.5901/mjss.2013.v4n10p80}

\begin{abstract}
Mentor programs are efficient, inexpensive and tailored ways of transferring knowledge from experts to less experienced employees. We put forward a conceptual model of affecting the knowledge transfer and creation in mentoring process and research numerically how the overlapping knowledge and the heterogeneous knowledge between mentor and mentee influence the performance of transfer activities, such as the quantity of the transferred knowledge and the increment of the created knowledge. We find that the moderate overlapping knowledge and heterogeneous knowledge between mentor and mentee are very important for the enhancement of the performance of transfer activities, that is to say the more transferred knowledge from mentor to mentee, the more effective knowledge creation becomes. The findings suggest a number of key factors that can affect knowledge transfer success, with suggestions for making a good pair of mentor-mentee and knowledge management. The authors would like to thank the Humanities and Social Sciences Research Fund of Ministry of Education of China under Grant No. 10YJA630082 for the financial supports.
\end{abstract}

Keywords: mentoring process; knowledge transfer; overlapping knowledge; heterogeneous knowledge; Disseminative capability; Absorbing capability

\section{Introduction}

Just as technological innovation up until the 1960s was treated as an unexplained variance in economic growth and performance, knowledge as an element within technological innovation has, until recently, been seen in a similar way. In order to keep the competitive advantages of companies, activities transferring and sharing knowledge, especially for tacit knowledge, have developed recently in multi-nationality companies. The interest in tacit knowledge has grown rapidly as it has become increasingly acknowledged that the contribution of knowledge innovation to performance of companies is not just simply associated with embodied knowledge, but is also highly dependent on disembodied, intangible assets and working practices[1]. Therefore, many companies have big interest in seeking to transfer some of the insights and best practices learned to their other divisions or other employee.

Research on knowledge transfer has developed out of studies focused on how companies could best accomplish technology transfers internally to keep or raise their competition ability and inter-company governance modes, including transfers in alliance settings [2]. Early work focused on the role of administrative structures on knowledge flows. It is found that companies with organizational structures that supported combining activities and sharing resources across 
subsidiary boundaries were more innovative[3]. Late work focused on the model of knowledge transfer and creation, such as the SECI process[4], knowledge creation through the conversion of tacit and explicit knowledge. Based the technology transfer and innovation literature, successful knowledge sharing can promote companies to master and get into practice product designs, manufacturing processes, and organizational designs that are new to them[5], and knowledge transfer is seen as occurring through a dynamic learning process where individuals of company continually interact each other to innovate or creatively imitate [6]. From this perspective, knowledge transfer involves the re-creation of a source's knowledge package in the recipient and mentoring process essentially is one of dynamic learning processes in which knowledge, embedded in many different structural elements of an organization, such as in the people and their skills, the technical tools, and the routines of company, could be transferred from junior colleague to senior.

The concept of mentoring is not new because it is closely related to craft apprenticeship schemes of the past[7]. Recently the topic about mentoring program and its importance not only to employees' career and professional development but also to transferring and sharing knowledge of companies have received substantial attention in the literature. Research has consistently demonstrated that those who are mentored reap significant benefits, such as career advancement, career satisfaction[8-11] and the knowledge embedded in individuals and organizational routines and best practices of company can also be imparted and inherited successfully. Mentoring provides a variety of developmental functions[12]

Mentoring programs are important mechanisms for transferring and creating knowledge by face-to-face knowledge exchanges. In mentoring programs of companies, mentors pass on their knowledge such as experiences, skills, techniques, crafts and know-how to mentees by saying and doing for them to absorb and grasp the knowledge through imitating and practicing and at the same time, create new knowledge[13-14]. Therefore the organizational distance and physical distance[15] between mentor and mentee is small. However, it is find that knowledge transfer to be challenging and knowledge sharing at companies proved to be like more difficult than expected[16-17]. Research on factors affecting knowledge transfer in mentoring process is advantageous to support knowledge exchange and to improve the performance of mentoring program.

\section{Factors Affecting Knowledge Transfer in Mentoring Process}

In mentoring program, though mentor can sometime learn from the mentee, mentor is still the main knowledge sender and mentee is the main recipient. Based on the researches mentioned above and literatures[18-21], we are inclined to consider the factors affecting knowledge transfer in mentoring process as three contextual domains: objects of knowledge transfer, such as overlapping knowledge and heterogeneous knowledge, subjects in knowledge transfer, such as capabilities of mentor and mentee, and learning culture of organization. Fig.1 presents a model of knowledge transfer that includes six key factors affecting knowledge transfer.

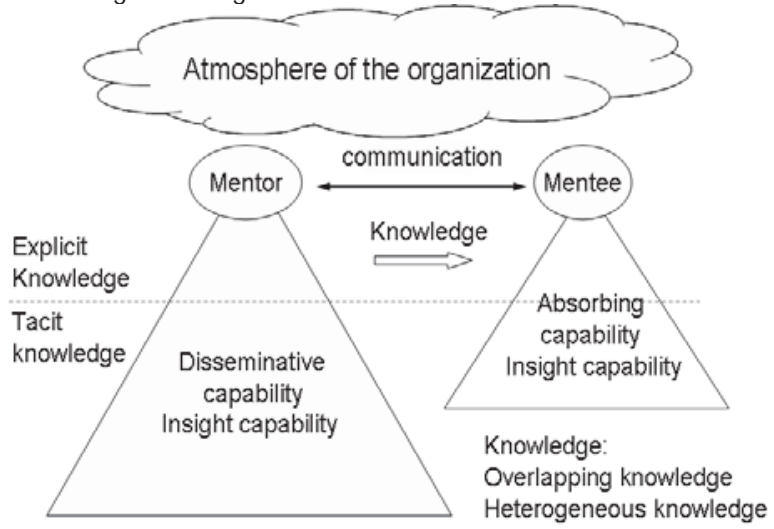

Figure 1. The model of affecting knowledge transfer in mentoring program

Under the atmosphere of mentoring environment, mentee generally will actively take part in mentoring program, while mentors' level of participating in is related to the their willingness of sharing knowledge with mentee and the difficult degree of transferring knowledge and the capabilities of transfer activities between mentor and mentee. 


\subsection{Disseminative capability of mentor}

Whether mentor is willing to share knowledge with mentee, the amount of knowledge provided, and the way used by mentor to impart his or her knowledge will directly influence the performance of knowledge transferring in mentoring process. There is significant evidence that effective re-creation requires that the knowledge package is made accessible to or de-contextualized for the recipient so that the recipient can convert it, adapt it or reconfigure it to its localized needs [22].In order to let the mentee understand the knowledge imparted the mentor should express the intended teaching objective clearly by utilizing tools such as body language and demonstration because knowledge, especially tacit knowledge, is hard to transfer from person to person. Mentor's willingness and capability to transfer knowledge is considered as disseminative capability[23], shown as a in our research. Disseminative capability is the one of the most influential factors effecting knowledge creation. We define a as the ratio of successfully disseminated knowledge quantity to total knowledge quantity the mentor have. The absorbing power describes the will of mentee

\subsection{Absorptive capability of mentee}

Absorptive capability describes the will and initiative of mentee to learn from mentor in mentoring process, the capability of mentee to comprehend the knowledge of the mentor's induction and combine with the previous knowledge he or she has had. We define absorptive capability as the ratio of the quantity of the knowledge absorbed successfully to the quantity of the knowledge disseminated by mentor, shown as parameter $\beta$.

\subsection{Insight capability of knowledge-creating}

Insight is an essential form of creative thinking. In mentoring process, both mentor and mentee may have a sudden inspiration while disseminating or absorbing knowledge and as a result new knowledge is created. This kind of knowledge creating is called insight knowledge-creating. From researches mentioned above, we think knowledge transfer in mentoring process also involves a dynamic learning and re-creation of mentor's knowledge package in mentee. Compared with mentor, mentee benefit more and get more inspiration by insight learning and learning by performing in transfer activities and hence mentee is more susceptible to enlightening. Therefore mentee's insight capability, shown in parameter $\gamma$, is more important for the knowledge increment to be produced in mentoring process. We define $y$ as the ratio of the quantity of the knowledge produced by enlightenment mentee received to the quantity of mentee's knowledge. Parameter $y$ represents for the insight capability of mentee.

\section{4 overlapping knowledge and heterogeneous knowledge}

Although Mentor and mentee usually have certain common knowledge because of similar major or job area, they have more difference in knowledge stock due to difference in backgrounds and working experiences. Whether mentee could understand and absorb the knowledge disseminated from mentor well is associated with the quantity of common knowledge they have. It has being proven in literatures that the heterogeneous knowledge in team work is good for arousing team creativity, but easy to cause opinion conflicts and communication problems. We use the parameter $\varepsilon$ to express the proportion of overlapping knowledge in the whole knowledge package of mentor and mentee and the parameter $k$ to express the ratio of heterogeneous knowledge between mentor and mentee.

\subsection{Learning culture}

Learning culture of a company is an important organizational environment factor which influences knowledge transfer and creation in mentoring process. Learning culture, such as advocating communication, encouraging innovation, tolerating mistakes and valuing talent, is helpful for making mentor and mentee exchange and share knowledge information and thus provides a good atmosphere for knowledge transfer and innovation. Learning culture makes norm distance shorten between mentor and mentee. We think the communication frequency between mentor and mentee can be used to measure the learning environment of the organization. In our research the communication frequency is represented as $\mu$. The higher communication frequency, the higher the level of transfer activity is. In fact, every time the knowledge exchange happened between mentor and mentee, some knowledge is transferred effectively from mentor to mentee. The higher the communication frequency is, the more quantity of knowledge is transferred and created. 
Parameter $\mu$ has great influence on the performance of transfer activities in mentoring program.

In conclusion, the influential factors of knowledge transfer and creation in mentoring process and the parameters defined are listed in table 1.

Table 1. Factors affecting knowledge transfer in mentoring process

\begin{tabular}{|c|c|c|}
\hline \multicolumn{3}{|c|}{ Influence factors } \\
\hline Subjects in Knowledge transfer & Objects in knowledge transfer & Learning culture \\
\hline Mentor's disseminative capability $(\alpha)$ & Proportion of overlapping knowledge & \multirow{3}{*}{$\begin{array}{l}\text { Communication } \\
\text { frequency }(\mu)\end{array}$} \\
\hline Mentee's absorptive capability $(\beta)$ & $(\varepsilon)$ & \\
\hline Mentee's insight capability $(\gamma)$ & $\begin{array}{l}\text { Ratio of heterogeneous knowledge } \\
\qquad(k)\end{array}$ & \\
\hline
\end{tabular}

\section{Models and Simulation}

Based on the factors summarized above, we research numerically the influence on the performance of knowledge exchange by measuring the quantity of the transferred and created knowledge.

\subsection{Variable definition}

We define the knowledge quantity $V_{0}$ before the knowledge exchange as the union set of mentor's and mentee's knowledge, that is, $V_{0}=$ quantity of mentor's knowledge + quantity of mentee's knowledge - quantity of overlapping knowledge.

We also define the ratio of mentor's tacit knowledge to his or her total knowledge as $\theta$. As tacit knowledge is far more than explicit knowledge for a person, we suppose $\theta \in(0.9,1)$.

The mentor's heterogeneous knowledge ratio is represented as $\omega_{1}$ and mentee's as $\omega_{2}$, so $\omega_{1}+\omega_{2}+\varepsilon=1$.

The heterogeneous knowledge ratio between mentor and mentee is represented as $k$ and $k=\omega_{1}: \omega_{2}$.

The relation of $\omega_{1}, \omega_{2}$ and $\varepsilon$ is shown in figure 2 .

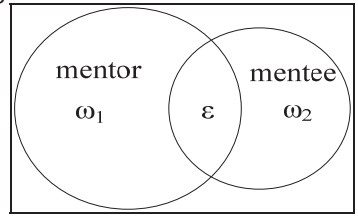

Figure 2. The knowledge ratio of mentor and mentee

In the process of mentoring program, as mentee absorbs and acquires the knowledge from mentor continuously, mentee's knowledge stock level increases gradually and the overlapping knowledge ratio $\varepsilon$ increases. This means that $k$ diminishes gradually, as shown in Figure 3 . Therefore, $k$ is always no less than one during the period of mentoring programs.

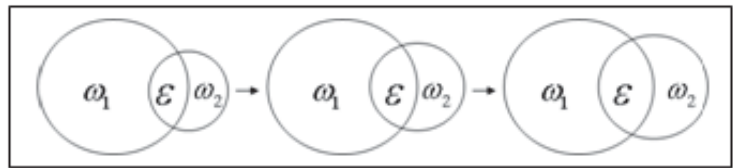

Fibure 3. The changing of knowledge ratio in a period of mentoringMathematical models of transferred and created knowledge quantity

We are intent on the research of the performance of one knowledge transfer by measuring the quantity of the transferred and created knowledge because the total performance is the sum.

According to the factors and variable definitions mentioned above, the quantity of the knowledge disseminated by mentor can be expressed as $V_{0} \theta \omega_{1} \alpha$ and the quantity acquired by mentee as $V_{0} \theta \omega_{1} \alpha \beta$, which is the knowledge transferred in the process of mentoring program. We let $V_{t f}=V_{0} \theta \omega_{1} \alpha \beta$. Then, mentee's total knowledge is equivalent to $V_{0}\left(\varepsilon+\omega_{2}\right)+V_{0} \theta \omega_{1} \alpha \beta$. Because mentee's insight capability is based on his or her total knowledge, the quantity of the 
enlightened knowledge is $\left[V_{0}\left(\varepsilon+\omega_{2}\right)+V_{0} \theta \omega_{1} \alpha \beta\right]^{\star} \gamma$. As insight capability is the main source of knowledge creation between mentor and mentee, the quantity of increased knowledge can be expressed as $\Delta V=\left[V_{0}\left(\varepsilon+\omega_{2}\right)+V_{0} \theta \omega_{1} \alpha \beta\right]^{\star} \gamma$.

As mentioned above, the mathematical models of transferred and created knowledge can be represented in formula (1) and (2):

$$
\begin{aligned}
& V_{t f}=V_{0} \theta \omega_{1} \alpha \beta \\
& \Delta V=\left[V_{0}\left(\varepsilon+\omega_{2}\right)+V_{0} \theta \omega_{1} \alpha \beta\right]^{\star} V
\end{aligned}
$$

\subsection{Basic conditions and hypotheses}

In the models above, if mentor and mentee are certain, the influence caused by $V_{0}, \theta$ and $\gamma$ would be certain. Thus we give them a fixed value:

1) $V_{0}=1, \theta=0.95$.

2) In another research[24], it is indicated that the knowledge creation rate of a node in a knowledge creation network obeys uniform distribution $U[0,0.1]$, so we let $\gamma=0.1$.

3) In the literature [25], it is pointed out that the knowledge disseminative capability and absorptive capability both obey the random distribution Rand[0,1]. Therefore we let the $\alpha \beta$ obey the distribution Rand[0,1] and increase with $\varepsilon$ because mentor's disseminative capability and mentee' $s$ absorptive capability both have positive correlation with overlapping knowledge ratio, that is $\alpha \propto \varepsilon, \beta \propto \varepsilon$.

\section{The Results}

The results achieved from simulating numerically, the effect of heterogeneous knowledge ratio $k$ and overlapping knowledge ratio $\varepsilon$ on the performance of knowledge transfer and creation, are shown in Fig.4 and Fig.5.

\section{1 the impact of $\varepsilon$ upon the quantities of transferred and created knowledge}

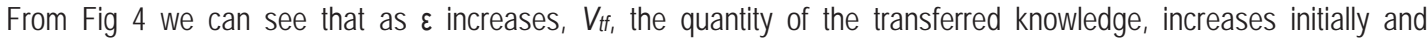
decreases gradually, and the maximum occurs while $\varepsilon$ is between 0.4 and 0.6 . This result verifies Yang's opinion[26], that is, the overlapping knowledge is the base for knowledge transfer. Enhancing knowledge exchange frequency, which is associated with the relationship between mentor and mentee, is propitious to the increase of overlapping knowledge. Increasing of overlapping knowledge between mentor and mentee is helpful for mentee to comprehend the knowledge transferred from mentor deeply and thus the efficiency of the knowledge transfer and creation will be improved. However, as the overlapping knowledge increases, the heterogeneous knowledge between mentor and mentee decreases. This situation would cause strong dependency between knowledge sender and receiver and also decrease knowledge transfer performance and organization's learning capability[27]. The results of our numerical research indicate that moderate amount of overlapping knowledge is most helpful for knowledge transfer besides the capabilities of mentor and mentee in transfer activities and the characteristics of knowledge. From Fig 5 we can see when $k$ is a constant, the quantity of knowledge created increases with $\varepsilon$ and the more the $\varepsilon$ is, the more the knowledge increment created by mentee's enlightenment is. It is indicated that there is a positive correlation between the knowledge increment $\Delta V$ and the overlapping knowledge $\varepsilon$. We can also see while $\varepsilon<0.4, \Delta V$ increases very quickly and while $\varepsilon>0.6, \Delta V$ increases very slowly in Fig 5 . There is almost the same space between increase and decrease as $V_{t f}$ coincidentally. The results hinted that the quantity of knowledge transferred from mentor to mentee has positive correlation with the knowledge increment created and also is important for enlightened knowledge.

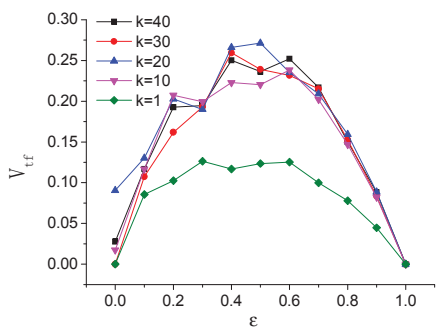


Figure 4. the effects on knowledge transfer

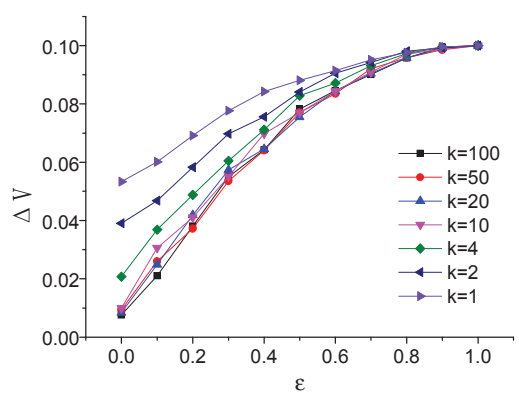

Figure 5. The effects on knowledge creation

\section{2 the impact of $k$ upon the quantities of transferred and created knowledge}

From Fig 4 we can see along with the increase of $k$ the quantity $V_{t f}$ of the knowledge transferred from mentor to mentee increases initially and decreases gradually. It is considered in our research that too large $k$ would bring an obstacle to communication and too small $k$ would affect the relationship between mentor and mentee while in the process of mentoring. This indicates that besides the moderate overlapping knowledge, the sufficient ratio of the heterogeneous knowledge quantity between mentor and mentee is also needed in order to promote knowledge transferring effectively. From Fig 5 we can see while $\varepsilon$ is fixed, the created knowledge quantity $\Delta V$ decreases while $k$ increases and the larger $k$ becomes, the less the knowledge increment be enlightened. When $k>=10$, it has little impact on $\Delta V$. And when $k$ increases to a certain level, the mentee's knowledge is very little compared with the mentor's so that the change of $k$ could barely make an impact on $\Delta V$. This is for the reason that the knowledge mentee have is too little for him or her to understand deeply the knowledge transferred by mentor and thus to create knowledge. These results hint us that if there are too big difference of knowledge stock level between mentor and mentee, the performance of transfer activities is not good.

\section{Conclusions and Suggestions}

Based on our researching numerically, the following conclusions can be reached:

1) The moderate $k$ and $\varepsilon$ is very important for knowledge transferring in mentoring process because $k$ and $\varepsilon$ can reflect the knowledge distant[15] between mentor and mentee. The knowledge distance is too big or too small all not good for transfer success.

2) In the mentoring process the quantity of the knowledge increment created is positive correlation with the quantity of the transferred knowledge from mentor to mentee.

3) Mentee's amount of knowledge stock is very important for own insight capability and its increase is positive correlation with the quantities of knowledge transferred and enlightened knowledge increment.

These findings will help company to put mentoring programs in practice effectively. A successful mentoring relationship relies on a reciprocal exchange between the mentor and mentee, in that the developmental experience involves responsibility and effort of both parties[24]. Based on our research, there are some suggestions for companies in areas of making mentor and mentee pair, knowledge management, such as knowledge sharing, transferring and creating, mentoring relationship supporting in mentoring programs:

1) The mentee should have a certain quantity of basic knowledge so that he or she has enough knowledge to understand, absorb and thus create knowledge and should throw himself or herself into mentoring program, such as being active in asking, hearing, learning by doing and etc.

2) Besides being willing in the mentor process mentors should be good at expressing their tacit knowledge in some ways because the tacit knowledge is hard to transfer and is the basis of new knowledge creation for organization.

3) It is necessary that between mentor and mentee there should be a moderate difference of knowledge stock level, which can be oriented from their working experience, positions, etc, and the moderate overlapping 
knowledge, which can be resulted from the similarly of background and their work, positions, other things in common and so on.

4) Learning culture is important to the success of mentoring programs for company because the subjects of mentoring process are the most important factors of the influence on performance of transfer activities. So it is necessary for company to build a good atmosphere of organization for knowledge exchange and innovation, such as communication for exchange and share knowledge information without any misgiving and tolerating mistakes between mentor and mentee and encouraging innovation. And further the performance of participation in mentoring programs could be related with employees' performance management to inspire employees to take part in the program and prompt mentors' willingness to share knowledge with mentees.

\section{References}

D. R. Charles \& J. R. Howells, Technology in Europe: Public and private Networks(Chichester, Wiley, 1992), P.3

Simonin, B.L., 1999. Transfer of marketing know-how in international strategic alliances: an empirical investigation of the role and antecedents of knowledge ambiguity. Journal of International Business Studies 30 (3), 463-490.

Birkinshaw, J.M., Morrison, A.J., 1995. Configurations of strategy and structure in subsidiaries of multinational corporations. Journal of International Business Studies 26 (1), 729-753.

Nonaka I, Takeuchi H. The Knowledg-creating Company. Oxford, England: Oxford University Press, 1995

Nelson, R. (Ed.), 1993. National Innovation Systems: A Comparative Analysis. Oxford University Press, New York.

Kim, L., Nelson, R.R., 2000. Technology, Learning, and Innovation: Experiences of Newly Industrializing Economies. Cambridge University Press, Cambridge, UK.

Wilbur, J. (1987). Does mentoring breed success?'. Training and Development Journal, 41, 28-41

Fagenson, E. A. (1988). The power of a mentor. Groups \& Organization Studies, 13, 182-194.

Kram, K. E. (1985). Mentoring at work: Developmental relationships in organizational life. Glenview, IL: Scott, Foresman.

Scandura, T. A. (1992). Mentorship and career mobility: An empirical investigation. Journal of Organizational Behavior, 13, 169-174.

Turban, D. B., \& Dougherty, T. W. (1994). Role of prote'ge' personality in receipt of mentoring and career success. Academy of Management Journal, 37, 688-702.

Kram, K. E. (1985). Mentoring at work: Developmental relationships in organizational life. Glenview, IL: Scott, Foresman.

Kram, K E. Improving the mentoring process[]]. Training and Development Journal, 1985, 39 (1): 16-23.

Chao, G T, Walz, P M and Gardner, PD. Formal and informal mentorships: A comparison on mentoring functions and contrast with nonmentored counterparts[J]. Personnel Psychology, 1992, 45(3): 619-636.

Cummings J L, Teng B S. Transferring R\&D knowledge: The key factors affecting knowledge transfer success [J]. Journal of Engineering and Technology Management, 2003, (20): 39-68.

Kerwin, K., Woodruff, D., 1992. Can olds hitch its wagon to Saturn's star? Business Week, 74.

Gupta, A.K., Govindarajan, V., 1991a. Knowledge flows and the structure of control within multinational corporations. Academy of Management Review 16 (4), 768-792.

Allen, T D, Poteet, M L, Russell, J E A, and Dobbins, G H. A field study of factors related to supervisors' willingness to mentor others[]]. Journal of Vocational Behavior, 1997, 50(1): 1 22.

Armstrong, S J, Allison, C W, and Hayes, J. Formal mentoring systems: An examination of the effects of mentor/protégé cognitive styles on the mentoring process[]]. Journal of Management Studies, 2002, 39(8): 1111 1137.

Lankau, M J, Riordan, C M, and Thomas, $\mathrm{C} \mathrm{H}$. The effects of similarity and linking in formal relationships between mentors and protégés[J]. Journal of Vocational Behavior, 2005, 67(2): 252 265.

Ragins, B R, Cotton, J L, and Miller, J S. Marginal mentoring: The effects of type of mentor, quality of relationship, and program design on work and career attitudes[]]. Academy of Management Journal, 2000, 43(6): 1177 1194.

(Devadas and Argote, 1995; Dixon, 1994; Leonard-Barton, 1988; Moreland et al., 1996)

Ragins, B. R. (1997). DiversiWed mentoring relationships in organizations: A power perspective. Academy of Management Review, 22, $482-521$

Tian Hui-min. Research on modeling and simulation of knowledge growth in research and development teams[D]. Nanjing: Nanjing University of Aeronautics and Astronautics, 2006. (in Chinese)

Tang Fang-cheng, Xi You-min. Knowledge transfer and dynamics behavior pattern of network organization ( II ): Absorptive capacity and disseminative capacity[J]. System engineering theory and practice, 2006, (9): 83-89.(in Chinese)

Yang Yu-bing, Pan An-cheng. Study on the relationship of strong tie network, overlapping knowledge, knowledge transfer[]]. Studies in Science of Science, 2009, 27(1): 25 29. (in Chinese)

March J G, Simon H A. Organizations[M]. New York: John Wiley, 1958. 